IRTF STUDIES OF THE IMPACTS OF COMET P/SHOEMAKERLEVY 9 INTO JUPITER

M. F. A'Hearn (U. Maryland.), K. Baines (JPL), D. Deming (NASAGSFC), T. Dowling (MIT), J. Goguen (JPL), C. Griffith (Northern Ariz. Univ.), H. Hammel (MIT), W. Hoffman (U. Ariz.), D. Hunten (U. Ariz.), D. Jewitt (U. Hawaii), T. Kostiuk (NASA-GSFC), S. Miller (Univ. College, London), K. Noll (STScI), G. Orton (JPL), K. Zahnle(NASA Ames)

The IRTF carried out a wide variety of programs related to the impacts of comet $\mathrm{P} /$ Shoemaker-Levy 9 into Jupiter. Images of the comet showed that fragment $K$ had faded to invisibility in the $J$ and $K$ bandpasses a week before impact but there is a tentative detection of a feature that may be a much brighter fragment $\mathrm{K}$ a few hours before impact.

Effects due to fragments $B$ and $F$ were undetectable near the limb of Jupiter using the MIRAC2 (Mid-InfarRed Array Camera 2 - built by the University of Arizona and the Smithsonian Astrophysical Observatory) at the same wavelengths used for studies of the effects of fragments $C, G$, and $R$. Lightcurves were obtained for the impact sites of fragment $\mathrm{C}$ at $2.248 \mu \mathrm{m}$, of fragment $G$ at several wavelenghts severely limited by fog, and of fragment $R$ at $7.85,10.3$, and $12.2 \mu \mathrm{m}$. The emission at site $\mathrm{R}$ is thermal in origin although it may not have a black-body spectrum. Observations at $7.85 \mu m$ (dominated by $\mathrm{CH}_{4}$ lines) show that the stratosphere above several sites was several $\mathrm{K}$ warmer than elsewhere one rotation after the impacts. The characteristic cooling time is about 2 days. Observations at wavelengths sensitive to the 150 to 400 mbar pressure levels showed several $K$ enhancements, although site E was barely detectable. At $400 \mathrm{mbar}$, the temperature excess disappeared rapidly while at $150 \mathrm{mbar}$ it remained visible for two weeks at site $\mathrm{L}$.

Emission by $\mathrm{NH}_{3}$ was strong over the impact sites with a different morphology than the thermal emission. The $\mathrm{NH}_{3}$ is estimated to be at the 1 to $30 \mathrm{mbar}$ level. $\mathrm{PH}_{3}$ was either depleted or significantly warmed. A sharp emission line of $\mathrm{CO}$ was observed that is probably at the $10 \mu b a r$ level and which requires enhanced $\mathrm{CO}$ in the stratosphere.

Observations at $3.41,2.27$, and $2.10 \mu \mathrm{m}$ show that the particles in the clouds must be at an altitude corresponding to no more than a few mbar. The clouds are not visible at $1.60 \mu \mathrm{m}$, suggesting that their optical depth is small in the near infrared. The clouds above site $\mathrm{K}$ spread at a zonal speed of 6 $\mathrm{m} \mathrm{s}^{-1}$ to yield a feature roughly $44 \mathrm{deg}$ in longitude and $15 \mathrm{deg}$ in latitude by July 28. This implies a decreasing velocity field with altitude.

Prior to the impacts, the southern auroral zone had been slightly brighter 
than the northern one. On July 27 , the northern auroral zone was much ( 6 to 7 times) brighter than the southern zone. The North Polar Hot Spot disappeared during the impacts and reappeared on July 28.

\section{OVERVIEW OF OBSERVATIONS OF THE IMPACTS}

\section{F. A'Hearn (U. Maryland)}

To attempt an overview of the worldwide scientific results only a month after an unprecedented event and before any results have been published is foolhardy. Nevertheless, the remarkable willingness of observers to transmit their results electronically to the wide community of other observers both indicates a revolution in scientific cooperation and makes it possible to attempt an overview at a very early stage.

The first remarkable result is that there are no confirmed measurements of impact flashes reflected from the Galilean satellites. While many possible detections were reported, they are all susceptible to either poor signal-tonoise ratio or to interpretation as artifacts, particularly due to variations in the scattered light from Jupiter. It is now clear that the reflected flashes were faint and that any satisfactory claims of detection will require simultaneous detections at more than one site.

The observations from HST show unambiguously that solid particles are present in the plume above the site very soon after the impact since the shadow of Jupiter is seen on the plume, indicating that the plume is reflecting sunlight. This requires rapid recondensation of solids (within a few minutes of the eruption of the fireball) unless a way is found to preserve solid cometary material in the fireball without totally vaporizing it.

Observations from the Galileo spacecraft show outbursts for several fragments which last a bit less than a minute, with a very sharp rise and a somewhat slower decline. It is unclear whether these are observations of the bolide entering the atmosphere, the subsequent fireball rising in the atmosphere, or some combination of the two. Comparison of timing for fragment $\mathrm{H}$ between Galileo and Calar Alto yields a delay that is too short to be consistent with both a Galileo observation of the bolide and the upward velocities obtained in some simulations of the fireball.

The most dramatic Earth-based detections are in the infrared where the brightness was sufficient to saturate detectors on some telescopes. The emission is largely thermal emission, rather than reflected sunlight. Light curves for different events are quite dissimilar but this is due in large part to the varying geometry, with later impacts occurring successively closer to the limb. In 